\title{
Missed Skeletal Trauma Detected by Whole Body Bone Scan in Patients with Traumatic Brain Injury
}

\author{
Yongsik Seo, ${ }^{1}$ Kum Whang, ${ }^{1}$ Jinsu Pyen, ${ }^{1}$ Jongwook Choi, ${ }^{1}$ Joneyeon Kim, ${ }^{1}$ Jiwoong $\mathrm{Oh}^{2}$ \\ Department of Neurosurgery, Yonsei University Wonju College of Medicine, Wouju, Korea \\ Department of Neurosurgery, ${ }^{2}$ Yonsei University College of Medicine, Seoul, Korea
}

Objective : Unclear mental state is one of the major factors contributing to diagnostic failure of occult skeletal trauma in patients with traumatic brain injury (TBI). The aim of this study was to evaluate the overlooked co-occurring skeletal trauma through whole body bone scan (WBBS) in TBI.

Methods : A retrospective study of 547 TBI patients admitted between 2015 and 2017 was performed to investigate their cooccurring skeletal injuries detected by WBBS. The patients were divided into three groups based on the timing of suspecting skeletal trauma confirmed : 1) before WBBS (pre-WBBS); 2) after the routine WBBS (post-WBBS) with good mental state and no initial musculoskeletal complaints; and 3) after the routine WBBS with poor mental state (poor MS). The skeletal trauma detected by WBBS was classified into six skeletal categories : spine, upper and lower extremities, pelvis, chest wall, and clavicles. The skeletal injuries identified by WBBS were confirmed to be simple contusion or fractures by other imaging modalities such as X-ray or computed tomography (CT) scans. Of the six categorizations of skeletal trauma detected as hot uptake lesions in WBBS, the lesions of spine, upper and lower extremities were further statistically analyzed to calculate the incidence rates of actual fractures (AF) and actual surgery (AS) cases over the total number of hot uptake lesions in WBBS.

Results : Of 547 patients with TBI, 112 patients (20.4\%) were presented with TBI alone. Four hundred and thirty-five patients with TBI had co-occurring skeletal injuries confirmed by WBBS. The incidences were as follows : chest wall (27.4\%), spine (22.9\%), lower extremities $(20.2 \%)$, upper extremities $(13.5 \%)$, pelvis $(9.4 \%)$, and clavicles $(6.3 \%)$. It is notable that relatively larger number of positive hot uptakes were observed in the groups of post-WBBS and poor MS. The percentage of post-WBBS group over the total hot uptake lesions in upper and lower extremities, and spines were $51.0 \%, 43.8 \%$, and $41.7 \%$, respectively, while their percentages of AS were $2.73 \%, 1.1 \%$, and $0 \%$, respectively. The percentages of poor MS group in the upper and lower extremities, and spines were $10.4 \%, 17.4 \%$, and $7.8 \%$, respectively, while their percentages of AS were $26.7 \%, 14.2 \%$, and $11.1 \%$, respectively. There was a statistical difference in the percentage of AS between the groups of post-WBBS and poor MS $(p=0.000)$.

Conclusion : WBBS is a potential diagnostic tool in understanding the skeletal conditions of patients with head injuries which may be undetected during the initial assessment.

Key Words : Brain injuries, Traumatic · Fractures, Bone · Radionuclide image.

-Received : June 16, 2020 •Revised : July 15, 2020 •Accepted : July 22, 2020

- Address for reprints : Jiwoong Oh

Department of Neurosurgery, Yonsei University College of Medicine, 50-1 Yonsei-ro, Seodaemun-gu, Seoul 03722, Korea

Tel : +82-2-2228-2166, Fax : +82-2-393-9979, E-mail : nsojw@yuhs.ac, ORCID : https://orcid.org/0000-0001-6065-4821

This is an Open Access article distributed under the terms of the Creative Commons Attribution Non-Commercial License (http://creativecommons.org/licenses/by-nc/4.0) which permits unrestricted non-commercial use, distribution, and reproduction in any medium, provided the original work is properly cited. 


\section{INTRODUCTION}

In acute trauma cases, the main focus is prioritizing the lifethreatening issues with immediate treatments. Therefore, there is a higher incidences of missed fractures in trauma patients. For instance, the most common attributing factors for missed injuries are hemodynamic instability of the traumatized patients, altered mental states with head injury, low index of suspicion, inadequate clinical assessment due to errors of inexperience, and missing findings on plain X-ray films ${ }^{9,12,16,17)}$. Hence, there is a limitation of concomitantly evaluating the co-existing musculoskeletal injuries during the initial resuscitation and assessment. Previously, many studies have reported the missed bone fractures in polytrauma patients with high incidenc$\mathrm{es}^{2,4,13,15,20,21)}$. However, there is a few studies specifically addressing the combined skeletal trauma in patients with traumatic brain injury (TBI) ${ }^{6}$. Occult non-cranial bone fractures in TBI may become a hindrance to proper post-trauma rehabilitations or a cause of late treatments such as orthopedic surgery or a long-term sequelae due to delayed detection ${ }^{8,16,19)}$.

A whole body bone scan (WBBS) is one of the useful diagnostic techniques to assess the multiple number of traumatic lesions in the whole skeletal system without invasive technique $^{5,13)}$. As mentioned earlier, the combined skeletal trauma in patients with TBI can be overlooked due to their altered neurologic states. This study pursues to survey the prevalence and clinical significance of the co-occurring injuries of skeletal systems specifically in patients with TBI through the earlyphase analysis of their WBBS taken routinely on the 7th day of managing acute trauma.

\section{MATERIALS AND METHODS}

This study was approved by Institutional Review Board of Wonju Severance Christian Hospital (IRB No. CR 320016).

\section{Patient population}

Nine hundred and thirty-seven patients with TBI were admitted to the department of neurosurgery in our institution from January 2015 to January 2017. Among them, the following patients were excluded from the final analysis : 121 patients with severe injury, 99 patients who refused WBBS, 44 patients who were transferred to other departments within the same institution, and 126 patients who were discharged due to early mortality or transfer to different institutions. A retrospective review of 547 patients with TBI was conducted, and the routine WBBS information was analyzed. There were 380 men and 167 women. The mean age on admission was $57 \pm$ 19.20 years. On average, the routine WBBS was performed on the 7th day after the onset of trauma and this was the standard trauma protocol of evaluating the trauma patients admitted to the neurosurgical department. According to the Glasgow Coma Scale (GCS), the patients with GCS score below 9 were classified as severe TBI with poor mental state (poor MS) while the score between 9 and 12 were referred as moderate TBI. The GCS score of 13 or more was categorized a mild TBI patients with good mentality.

\section{Defining fractures by WBBS with follow-up im- aging modalities}

All the injuries of skeletal system detected by WBBS were radiographically reported in conjunction with the review of X-rays by two specialists from the department of nuclear medicine. With WBBS, the fractures were defined by an increased radionuclide tracer uptake which was intense to confirm bone injury. Weak signals of tracer uptake were considered to be 'bone bruise' or simple 'bone contusion ${ }^{33,18)}$. The definite fractures with hot uptake of the tracer were presumed to be due to recent trauma, and the missed lesions were followed by X-rays or computed tomography (CT) scans if necessary.

\section{Categorization of the injuries of skeletal system}

The skeletal trauma detected by WBBS was initially classified into six skeletal categories : spine, upper and lower extremities, pelvis, chest wall, and clavicles. The patients were divided into three groups based on the timing of suspecting skeletal trauma confirmed : 1) before WBBS (pre-WBBS); 2) after the routine WBBS (post-WBBS) with good mental state and no initial musculoskeletal complaints; and 3) after the routine WBBS with poor mental state (poor MS) (Fig. 1). To be more specific, the 'pre-WBBS' was defined as a group of patients with expressed painful symptoms or physical evidence of external wounds. The skeletal trauma of this group was clearly suspected prior to WBBS. On the other hand, the 'post-WBBS' was defined as a group of patients with skeletal injury diagnosed after WBBS because the patients showed 
neither definite clinical symptoms nor were presented with definite wounds associated with the skeletal injury. This particular group of patients were hospitalized to treat head trauma as a major injury. Because of the nature of trauma, the patients of post-WBBS were indicated for a routine WBBS, and the incidental hot uptake findings of the co-existing skeletal trauma were followed by X-rays or CT scans after WBBS, i.e., post-WBBS. Lastly, The 'poor MS' is a group of patients with altered consciousness as well as no definite external wounds. In other words, the skeletal injury was masked by poor mental states in these cases, thus, the diagnosis of skeletal injuries was possible when a routine WBBS was undertaken. The skeletal traumas of upper, lower extremities, and spine were detected and categorized according to the above patient groups.

However, the skeletal traumas of chest wall, pelvis, and clavicles could not be categorized into the above three groups of pre-, post-WBBS, or poor MS. The reason being that all the patients admitted to our hospital underwent routine trauma series including chest and pelvic X-rays. Thus, most of the fractures were discovered, diagnosed, and confirmed during the initial trauma assessment before WBBS regardless of the patients' clinical symptoms. The co-occurring injuries of chest

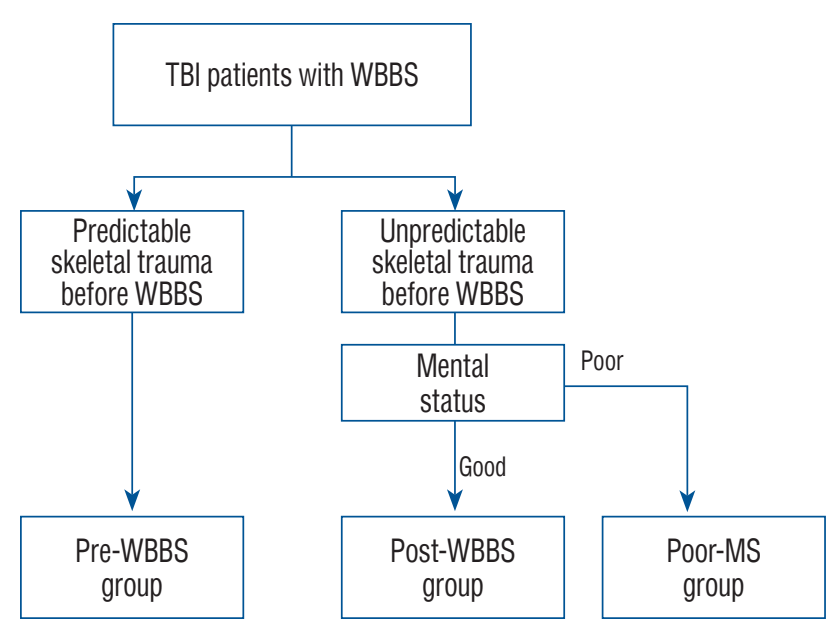

Fig. 1. Categorization of the patients based on the timing of suspecting skeletal trauma in patients with TBI. When the patient was presented with positive findings of symptoms (e.g., pain) or signs (e.g., external wound) in the skeletal system, this is referred as predictable skeletal trauma before WBBS is taken (pre-WBBS). Another group of patients were presented with no definite symptoms or signs of occult bone fractures, then this group is referred as 'post-WBBS' with unpredictable skeletal trauma before WBBS. Among this group of patients, if the patients were presented with altered consciousness with poor GCS scores, then the patients were referred as 'poor MS'. TBI : traumatic brain injury, WBBS : whole body bone scan, poor MS : poor mental status. wall, pelvis, and clavicles were investigated mainly through the epidemiologic analysis of their prevalence in patients with TBI.

\section{Clinical follow-up processes after WBBS}

Most of the lesions with an increased uptake of the radionuclide tracers in WBBS were confirmed by the follow-up plain X-ray films or CT scans to report actual fractures (AF). Physicians then decided the treatment plans to be either surgical or non-surgical with conservative management. Except for the chest wall lesions (i.e., due to the reasons mentioned above no follow-up X-rays or CT scans were undertaken after WBBS), all the other lesions with hot uptakes detected by WBBS were sorted out for an advanced analysis by calculating their incidence rates of becoming $\mathrm{AF}$, and their probability of becoming actual surgery (AS) cases : \% AF = (number of fractures / number of hot uptake lesions $) \times 100$; $\%$ AS $=$ (number of surgical cases / number of hot uptake lesions) $\times 100$.

\section{Imaging protocols}

Infinia Dual Gamma camera (GE Health Care, Chicago, IL, USA) model was used as a bone scan in our institution. No premedication was required for the examination, and the bone scan was performed 3-4 hours after the intra-venous injection of $99 \mathrm{~m}$ Tc-methylene diphosphonate $(740 \mathrm{MBq})$ on the day of the examination. Bone scan images (scan speed, $13 \mathrm{~cm} / \mathrm{min}$; matrix, $256 \times 256$ ) were obtained with a gamma camera equipped with a low energy, high resolution parallel hole collimator and stored in a computer system (Xeleris; GE Health Care). There were no special precautions other than urination and water intake before the examination.

\section{Statistical analysis}

All the studies were statistically analyzed using IBM SPSS version 23 (IBM, Armonk, NY, USA). Categorical variables were presented as frequencies and percentages. Data were all analyzed by chi-square tests and analysis of variance (ANOVA) with post-hoc Bonferroni's test. The statistical significance was confirmed when the $p$ value was $<0.05$.

\section{RESULTS}

Of 547 patients with TBI, who underwent routine WBBS, 
only 112 (20.5\%) did not show any skeletal injuries by WBBS, i.e., these are the patients with TBI alone. Based on the subclassification of the patients by their GCS scores, the number of patients with mild, moderate, and severe TBI were 420, 64, and 63, respectively. The total number of hot uptake lesions in WBBS in 547 patients were 994 (994/547, 1.82 lesions per patient). As shown in Table 1 , the most common radionuclide uptake lesion was chest wall $(n=273)$, followed by spine $(n=228)$. The percentage of AF over the total number of hot uptake lesions were ranging from $17 \%$ to $48 \%$. Moreover, the percentage of AS cases over the total number of hot uptake lesions were ranging from $4 \%$ to $38 \%$ as summarized in Table 2.

\section{Comparison of pre-, post-WBBS groups, and poor MS group in the lesions of upper, lower extremi- ties, and spines}

As we mentioned earlier, the trauma lesions of upper, lower extremities, and spine areas were not parts of routine trauma $\mathrm{X}$-ray series. Thus, the imaging of these lesions were investigated when the patients expressed the clinical symptoms or via physical examinations or through the routine advanced imaging modality such WBBS. According to the timing of suspecting the trauma lesions, the patients were sub-divided into three groups of pre-WBBS, post-WBBS, and poor MS. As it is stated in the method section in detail, the X-rays of chest including clavicles, and pelvis were in the standard trauma protocol in our institution, hence, an early diagnosis with appropriate management is usually immediately initiated after trauma.

Of all the hot uptake lesions detected by WBBS, 248 lesions were found in the pre-WBBS group while the same number (248 lesions) were detected in the post-WBBS group. WBBS detected 68 lesions in the patients of poor MS group. The \% AF (i.e., calculated from the ratio of number of fractures over the total number of hot uptake lesions) were as follows : preWBBS ( $n=93,37.5 \%)$; post-WBBS ( $n=17,6.85 \%)$; and poor MS $(n=17,25 \%)$ (Table 3). The \% AS cases (i.e., calculated from the ratio of number of surgeries over the total number of hot uptake lesions) were as follows : pre-WBBS ( $n=69,27.82 \%)$; post-WBBS ( $n=2,0.81 \%)$; and poor MS ( $n=11,16.18 \%)$. The relative risks (RR) of hot uptake lesions in WBBS to become true bone fractures are as follows : post-WBBS vs. pre-WBBS (RR, 0.18); and post-WBBS vs. poor MS (RR, 0.27) $(p<0.001)$ with statistical significance. On the other hand, the groups of pre-WBBS and poor MS did not show any statistically remarkable differences in $\% \operatorname{AF}(p=0.055$; RR, 1.5). The RR of hot uptake lesions in WBBS to become true surgical cases were 0.02 (post-WBBS vs. pre-WBBS) and 0.05 (post-WBBS

Table 1. Summary of the hot uptake lesions in whole body bone scan based on trauma types

\begin{tabular}{|c|c|c|c|c|c|c|}
\hline Type of accident & Upper extremities & Lower extremities & Spine & Pelvis & Chest wall & Clavicle \\
\hline Slip down (n=223) & $35(12.8)$ & $50(36.6)$ & $58(21.2)$ & $23(8.4)$ & $50(18.3)$ & $7(2.6)$ \\
\hline Traffic accident $(n=413)$ & $56(11.2)$ & $94(37.0)$ & $80(15.7)$ & $39(7.7)$ & $110(21.7)$ & $34(6.7)$ \\
\hline Falling down ( $n=173)$ & $23(11.5)$ & $27(27.0)$ & $48(24.0)$ & $12(6.0)$ & $47(23.5)$ & $16(8.0)$ \\
\hline Assault ( $n=11)$ & $1(11.1)$ & $2(44.4)$ & $1(11.1)$ & $1(11.1)$ & $2(22.2)$ & $4(8.0)$ \\
\hline Unknown (n=77) & $13(13.8)$ & $13(27.7)$ & $11(7.4)$ & $7(7.4)$ & $33(35.1)$ & $0(0.0)$ \\
\hline Others $(n=97)$ & $7(6.3)$ & $15(26.8)$ & $30(10.7)$ & $12(10.7)$ & $31(27.7)$ & $2(1.8)$ \\
\hline Total $(n=994)$ & $135(13.5)$ & $201(20.2)$ & $228(22.9)$ & $94(9.4)$ & $273(27.4)$ & $63(6.3)$ \\
\hline
\end{tabular}

Values are presented as number (\%)

Table 2. The percentage of actual fractures (\% AF) and actual surgical cases (\% AS) over the total number of hot uptake lesions detected by WBBS

\begin{tabular}{|c|c|c|c|c|c|c|}
\hline & Upper extremities & Lower extremities & Spine & Pelvis & Chest wall & Clavicle \\
\hline Total hot uptake lesion & 135 & 201 & 228 & 94 & 273 & 63 \\
\hline Number of fracture & $48(36.0)$ & $40(20.0)$ & $39(17.0)$ & $16(17.0)$ & N.A. & $30(48.0)$ \\
\hline Number of surgery & $35(26.0)$ & $25(12.0)$ & $22(10.0)$ & $4(4.0)$ & $0(0.0)$ & $24(38.0)$ \\
\hline
\end{tabular}

Values are presented as number (\%). WBBS : whole body bone scan, N.A., not applicable 
vs. poor MS) $(p<0.001)$. Again, there was no statistical difference between the groups of pre-WBBS and poor MS in the aspect of $\%$ AS ( $p=0.05 ; \mathrm{RR}, 1.71)$. It is noteworthy that the $\%$ AF and \% AS calculated to compared the post-WBBS and preWBBS groups showed a statistical significance while the same calculation and analysis between the groups of pre-WBBS and poor MS did not (Fig. 2). To sum up, the pre-WBBS and poor MS groups showed a higher incidence of fracture risks as well as an increased number of surgical cases of missed or occult bone fractures detected by WBBS according to our data and analysis.

Table 3. The number of hot uptake lesion and fracture and surgical case in each groups

\begin{tabular}{lccc} 
Lesion & $\begin{array}{c}\text { Hot uptake } \\
\text { lesion }\end{array}$ & Fracture & $\begin{array}{c}\text { Surgical } \\
\text { case }\end{array}$ \\
\hline $\begin{array}{l}\text { Upper extremities } \\
\text { Pre-WBBS }\end{array}$ & 55 & 36 & 30 \\
Post-WBBS & 65 & 5 & 1 \\
Poor MS & 15 & 7 & 4 \\
Lower extremities & 78 & 28 & 19 \\
Pre-WBBS & 88 & 6 & 1 \\
Post-WBBS & 35 & 6 & 5 \\
Poor MS & & & \\
Spine & 115 & 29 & 20 \\
Pre-WBBS & 95 & 6 & 0 \\
Post-WBBS & 18 & 4 & 2 \\
Poor MS & & & \\
\hline
\end{tabular}

Values are presented as number (\%). WBBS : whole body bone scan, poor MS : poor mental status

\section{Fractures of pelvis, chest walls including clavicles}

Hot tracer uptake lesions of pelvis were detected by WBBS in 94 patients (17.18\%). Of 94 lesions of skeletal trauma, the AF were found and diagnosed in 16 patients (17.02\%) while the actual surgeries of the fractures were proceeded in four patients (4.26\%). Two hundred seventy-three patients (49.9\%) showed hot uptake lesions in the chest walls. The radionuclide tracer uptakes of clavicles were found in 63 patients. Of 63 lesions, the AF were confirmed in 30 patients (47.62\%). The surgery was required to manage broken clavicles in 24 patients (38.1\%). None of these undetected skeletal trauma contributed to mortality of the patients.

\section{DISCUSSION}

This study demonstrated that missed skeletal trauma in patients with TBI is common and this finding reflects one aspect of the clinical difficulties in managing acute trauma. The retrospective review of the patient data provoked the authors that the probabilistic criteria of skeletal trauma specifically in TBI is urgently needed in simultaneously and efficiently assessing the patients when the head trauma is the major focus of management by most of the neurosurgeons at the emergency department. With references to the kinetic elements of the trauma in Vittel triage criteria, we attempted to sub-classify the sites of skeletal trauma in TBI into six groups for analysis (Table 1). Subsequently, the timing of suspecting skeletal injuries was further divided into three groups based on the time of WBBS taken (i.e., pre-WBBS, post-WBBS, and poor MS). We
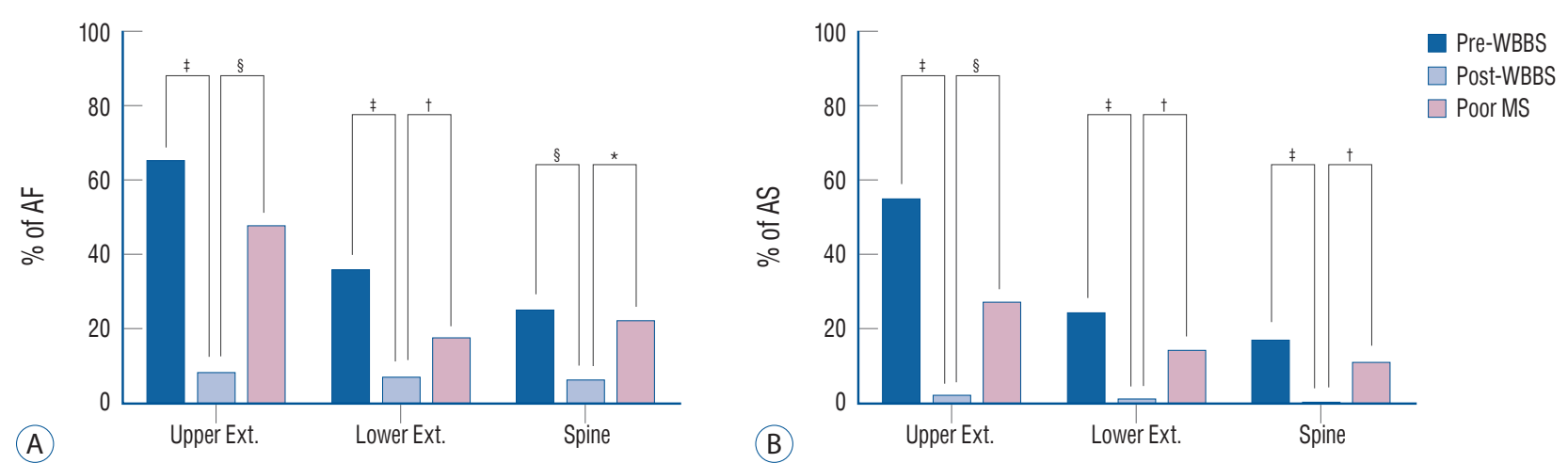

Fig. 2. Analysis of \% AF (A) and \% AS (B) in the upper (upper Ext.) and lower extremities (lower Ext.), and spines in the three groups of the patients (pre-, post-WBBS, and poor MS). Overall, there were significantly higher incidences of fractures and surgical treatments in pre-WBBS and poor MS groups when compared with post-WBBS group. However, there was no statistical significance in the pre-WBBS group and poor MS groups. ${ }^{*} p<0.05 .{ }^{\dagger} p<0.005$. ${ }^{\ddagger} p<0.001 .{ }^{\S} p<0.0005$. AF : actual fractures, AS : actual surgery, Ext. : extremity, poor MS : WBBS : whole body bone scan poor mental status. 
counted the number of AF and AS cases of the newly diagnosed lesions detected by WBBS. Briefly speaking, we proposed a simple percentage calculation of actual fracture rates (\% AF) and actual orthopedic or plastic surgical rates (\% AS) over the total number of positive radionuclide tracer uptakes of extra-cranial lesions detected by WBBS in TBI patients. The calculated parameters were used to investigate the prevalence of skeletal trauma in TBI.

According to our data, the patients of pre-WBBS are those who were alert enough to express their symptoms or are presented with open external wound, hence, the rapid assessments and early diagnosis with prompt management were delivered to the patients. Intuitively, the routine WBBS performed on the 7th day after trauma doubly confirmed that the hot tracer uptake lesions overlapped with the diagnosed trauma before WBBS, hence, they showed high \% AF and \% AS in our analysis especially in the lesions of upper, lower extremities, and spines. Akin to this finding, the patients of post-WBBS were those with high GCS with neither symptoms or signs of peripheral skeletal injuries and the \% AF and \% AS were low when compared independently with either pre-WBBS or poor MS. These comparisons were anticipated to be statistically significant. The degree of missed injuries of post-WBBS patients were mild that no new treatments were introduced during the hospitalization as we expected.

However, interestingly, the \% AF and \% AS between the pre-WBBS and poor MS groups showed no inter-group difference statistically. This analytical finding has many significances in terms of future trauma management of patients with severe TBI. The patients with poor MS showed as high $\% \mathrm{AF}$ and $\% \mathrm{AS}$ as the patients with pre-WBBS. To be more clear and concise, the hot uptake lesions of missed bone fractures detected by WBBS in the unconscious patients with poor GCS are similar to the incidence of fractures diagnosed in patients with good GCS. The physical impact of trauma can be severe enough to cause diminished consciousness as well as profound injuries to other parts of the body. This result can show many implications. Most importantly, the key lesson from this study is that there are number of different groups of TBI patients who are at increased risks of having injuries missed or undetected on initial presentation. Many studies have reported that TBI can be combined with an increased risk of missed skeletal trauma ${ }^{5,8,19)}$. We strongly believe that our patients in the 'poor MS' group are one group that may largely benefit from early WBBS due to the routine standard trauma protocol in our institution, because the fractures identified involving long bones (in the upper and lower extremities), spine, and clavicles often required a change in the treatment plans (e.g., delayed surgery) in this specific TBI group according to our data. Owing to the nature of physical trauma in general, the patients with TBI may not always present head injury alone. Therefore, it is crucial that we have to continuously improve the errors of medical assessment to reduce the rates of clinically significant missed trauma and their subsequent complications in the skeletal system of patients with TBI. Wei et al. ${ }^{22)}$ reported that the rate of missed distal bone fracture when using plain X-ray films is up to $7.6 \%$ in TBI. In an attempting to lower this incidence of missed injuries, Huang et al. ${ }^{10)}$ conducted a clinical study with whole body X-rays with Lodox/ Statscan for early detection of commonly missed trauma in TBI. Their institutional data showed that the missed extracranial injuries in TBI were up to $39.1 \%$ while the whole body $\mathrm{X}$-rays reduced this rate of missed bone fractures down to $1.6 \%$ with low cost and low radiation exposure. This is an interesting study but we would like to argue that the bone fractures are usually detected with the requirements of $30-50 \%$ of inorganic changes of the affected bones. Whereas WBBS can detect the lesions of skeletal trauma with only $3-5 \%$ of bone changes $^{1,23)}$.

Many previous reports discussed the missed injuries in pediatric TBI and spinal cord injury ${ }^{7,8,19,20)}$. However, one must consider a unique characteristics of pediatric skeleton (e.g., presence of growth plates) so the pediatric cases were out of our scope in this specific study. To our knowledge, this study is the first clinical article to report the analysis of the epidemiology of combined skeletal trauma in adult patients with TBI based on the data obtained from WBBS performed during the early stage of managing acute trauma. One part of the legacies in this study was to aid clinical decision making concerning the optimal management of occult skeletal trauma in patients with TBI. The consequences of delayed diagnosis of occult bone fractures, especially in long bones, will directly affect the quality of clinical recovery of the patients due to a constant pain, prolonged hospitalization, and loss of general functions to rehabilitate. One of the highlighted findings in our study is the significance of high incidence of long bone fractures in the upper and lower extremities of the initially unconscious patients with TBI. With deficits in motor performances primar- 
ily originated from these undetected fractures will ultimately affect their functional outcomes ${ }^{6}$.

The limitations of this study are discussed as follows. This is a retrospective study by nature based on medical chart reviews. Insufficient physical examinations and incomplete medical recording would all contribute to the over-estimation of the inter-group analysis. However, all the data were rigorously analyzed with the two neurosurgeons and six neurosurgical nurses. The surgical indications for the hot uptake lesions of WBBS varied among the surgeons. In this study, we only considered the operable lesions to calculate the AS cases. Similar studies were introduced and discussed in this article and reported different results when compared with our data. For instance, Lee et al. ${ }^{14)}$ reported that co-existing injuries of pelvis and chest are more common in TBI. Whereas, our analysis showed that the combined injuries in the upper and lower extremities, spines, and clavicles are as prevalent as the thoracic in TBI. Janjua et al. ${ }^{11)}$ supported our data that the missed musculoskeletal injuries were more commonly seen in the extremities in the patients with TBI. Whether the study design is retrospective or prospective will also produce different analysis. The imaging modalities available and the timing of the assessment after the trauma are also variable determinants in the analysis. Therefore, randomized clinical trials from multiple trauma centers will be necessary to set up a general protocol of managing missed fractures in TBI.

Technical limitation is that a period of at least 48 to 72 hours after injury is required for a reliable bone scan with high sensitivity ${ }^{1}$. However, due to low specificity, the hot uptakes in WBBS do not always correspond to AF in the plain Xrays $^{18)}$. A radionuclide bone scan uses the metabolic activities of bones at a certain medical state. Thus, the abnormal activities by infection, tumor, degenerative changes, and simple bone contusion without fractures can also be detected as false positive lesions.

\section{CONCLUSION}

We analyzed the findings of WBBS in the patients of TBI. Our study showed that co-existing skeletal trauma was very common but easy to miss in TBI. Without routine WBBS, many bone fractures would have been overlooked. Therefore, we conclude that WBBS is a potential diagnostic tool in un- derstanding the skeletal conditions of patients with head injuries, especially unclear mental state, which may be undetected during the initial assessment.

\section{CONFLICTS OF INTEREST}

No potential conflict of interest relevant to this article was reported.

\section{INFORMED CONSENT}

This type of study does not require informed consent.

\section{AUTHOR CONTRIBUTIONS}

\author{
Conceptualization : JO \\ Data curation : YS, JC \\ Formal analysis : JO, JP \\ Funding acquisition : JK, KW \\ Methodology: JO, JP \\ Project administration : YS \\ Visualization : YS \\ Writing - original draft : YS \\ Writing - review \& editing : JO
}

\section{ORCID}

$\begin{array}{ll}\text { Yongsik Seo } & \text { https://orcid.org/0000-0002-6377-9838 } \\ \text { Kum Whang } & \text { https://orcid.org/0000-0002-3701-847X } \\ \text { Jinsu Pyen } & \text { https://orcid.org/0000-0002-8008-4122 } \\ \text { Jongwook Choi } & \text { https://orcid.org/0000-0003-2593-3870 } \\ \text { Joneyeon Kim } & \text { https://orcid.org/0000-0002-9407-0127 } \\ \text { Jiwoong Oh } & \text { https://orcid.org/0000-0001-6065-4821 }\end{array}$

\section{References}

1. Bauer GC: The use of radionuclides in orthopaedics. Radionuclide scintimetry of the skeleton. 1968. Clin Orthop Relat Res 287 : 3-12, 1993

2. Brooks A, Holroyd B, Riley B : Missed injury in major trauma patients. 
Injury $35:$ 407-410, 2004

3. Deininger HK : Skeletal scintigraphy as an addition to the roentgenological examination in traumatology (author's transl). Radiologe 21 : 3545, 1981

4. Enderson BL, Maull KI : Missed injuries. The trauma surgeon's nemesis. Surg Clin North Am 71 : 399-418, 1991

5. Frawley PA, Mills JA, Murton F, Ware R: Bone scanning in the multiply injured patient. Aust N Z J Surg 65 : 390-393, 1995

6. Garland DE, Bailey $S$ : Undetected injuries in head-injured adults. Clin Orthop Relat Res 155 : 162-165, 1981

7. George MP, Bixby S : Frequently missed fractures in pediatric trauma: a pictorial review of plain film radiography. Radiol Clin North Am 57 : 843-855, 2019

8. Heinrich SD, Gallagher D, Harris M, Nadell JM : Undiagnosed fractures in severely injured children and young adults. Identification with technetium imaging. J Bone Joint Surg Am 76 : 561-572, 1994

9. Hirshberg A, Wall MJ Jr, Allen MK, Mattox KL : Causes and patterns of missed injuries in trauma. Am J Surg 168 : 299-303, 1994

10. Huang HC, Fu CY, Hsieh CH, Wang YC, Wu SC, Chen RJ, et al. : Lodox/ Statscan facilitates the early detection of commonly overlooked extracranial injuries in patients with traumatic brain injury. Eur J Trauma Emerg Surg 38 : 319-326, 2012

11. Janjua KJ, Sugrue M, Deane SA : Prospective evaluation of early missed injuries and the role of tertiary trauma survey. J Trauma 44 : 10001007, 1998

12. Kremli MK : Missed musculoskeletal injuries in a university hospital in Riyadh: types of missed injuries and responsible factors. Injury 27 : 503506, 1996

13. Lee KJ, Jung K, Kim J, Kwon J : Bone scan as a screening test for missed fractures in severely injured patients. Orthop Traumatol Surg Res
$100: 953-957,2014$

14. Lee WC, Chen CW, Lin YK, Lin TY, Kuo LC, Cheng YC, et al. : Association of head, thoracic and abdominal trauma with delayed diagnosis of coexisting injuries in critical trauma patients. Injury 45 : 1429-1434, 2014

15. Mackersie RC, Shackford SR, Garfin SR, Hoyt DB : Major skeletal injuries in the obtunded blunt trauma patient: a case for routine radiologic survey. J Trauma $28:$ 1450-1454, 1988

16. Mrozek S, Gaussiat F, Geeraerts $T$ : The management of femur shaft fracture associated with severe traumatic brain injury. Ann Fr Anesth Reanim 32 : 510-515, 2013

17. Probst C, Pape HC, Hildebrand F, Regel G, Mahlke L, Giannoudis P, et al. : 30 years of polytrauma care: an analysis of the change in strategies and results of 4849 cases treated at a single institution. Injury $40: 77$ 83, 2009

18. Schmidt $C$, Deininger HK : The occult fracture in the roentgen picture and its detection using bone scintigraphy. Radiologe 25 : 104-107, 1985

19. Sobus KM, Alexander MA, Harcke HT : Undetected musculoskeletal trauma in children with traumatic brain injury or spinal cord injury. Arch Phys Med Rehabil 74 : 902-904, 1993

20. Soundappan SV, Holland AJ, Cass DT : Role of an extended tertiary survey in detecting missed injuries in children. J Trauma 57 : 114-118, 2004

21. Ward WG, Nunley JA : Occult orthopaedic trauma in the multiply injured patient. J Orthop Trauma 5 : 308-312, 1991

22. Wei CJ, Tsai WC, Tiu CM, Wu HT, Chiou HJ, Chang CY : Systematic analysis of missed extremity fractures in emergency radiology. Acta Radiol 47 : 710-717, 2006

23. Yang DC, Ratani RS, Mittal PK, Chua RS, Pate SM : Radionuclide threephase whole-body bone imaging. Clin Nucl Med 27 : 419-426, 2002 